\title{
Preparation and Evaluation of Palonosetron Hydrochloride Oral Thin Film
}

\author{
Sreebash Chandra Bhowmik ${ }^{1}$, Marzia Alam ${ }^{2}$ and Md. Saiful Islam Pathan ${ }^{1}$ \\ ${ }^{1}$ Department of Pharmacy, State University of Bangladesh, Dhanmondi, Dhaka-1209, Bangladesh \\ ${ }^{2}$ Department of Pharmacy, BRAC University, Mohakhali, Dhaka-1212, Bangladesh
}

(Received: May 14, 2019; Accepted: June 18, 2019; Published: July 22, 2019)

\begin{abstract}
The purpose of the current study was to develop a fast dissolving polymeric oral thin film containing palonosetron hydrochloride having good mechanical properties, fast disintegration, dissolution and good drug content uniformity. Solvent casting method was used to prepare the films. Compatibility between drugs and excipients were studied using FTIR and HPLC. Nine different formulations of film from F1 to F9 were prepared using different concentration of polymer A at drug-polymer A ratio (1:26), (1:28), (1:30), (1:32), (1:34), (1:36), (1:38), (1:40), (1:42) and at polymer A-plasticizer B of (65:10), (70:10), (75:10), (40:10), (42.5:10), (45:10), (47.5:10), (50:10), (52.5:10), respectively. The in vitro dissolution study was carried out in phosphate buffer $(\mathrm{pH} 6.8)$ at $37 \pm 0.5^{\circ} \mathrm{C}$ and $50 \mathrm{rpm}$ using USP XXIV paddle method. Physicochemical evaluations of all the batches were performed including weight variation, thickness, folding endurance, $\mathrm{pH}$, in vitro disintegration and drug release, FTIR and content uniformity test. Maximum and minimum drug dissolution were found in F6 (108.7\%) and in F1 (98.5\%), respectively. The maximum and minimum disintegration time were in F9 (43.8 sec) and F1 (25 sec), respectively which demonstrated that disintegration of the film was directly proportional to the polymer A and plasticizer B concentration. It is quite evident from the present research work that the film prepared using polymer A-plasticizer B were smooth, mechanically strong and easy to peel out. Among all the batches, formulation F5 showed best results with respect to disintegration (33 sec), drug dissolution (105\%), content uniformity $(98.51 \%)$ and folding endurance (731). Therefore, it can be said that combination of polymer A and plasticizer B can be prospectively used for the preparation of palonosetron hydrochloride oral thin film.
\end{abstract}

Key word: Oral thin film, Palonosetron hydrochloride.

\section{Introduction}

Oral route of drug administration is the most preferred route due to its ease of administration, noninvasiveness, adaptability, patient compliance and acceptability. Regarding oral route of drug administration, many substitutes have continuously been presented by using recent novel technologies for pediatrics, geriatrics, nauseous and non-compliant patients (Arya et al., 2010). Orally disintegrating films (ODFs), when placed on tongue, immediately hydrates by soaking saliva following disintegration and dissolution releasing active pharmaceutical agent from the dosage form (Chauhan et al., 2012).
ODFs are special kind of formulations which are commonly prepared using hydrophilic polymers enabling rapid dissolution upon contact with saliva. Oral disintegrating tablets (ODTs) and oral disintegrating films (ODFs) are the typical examples of orally disintegrating drug delivery systems (Dixit et al., 2009). The administration of medications to pediatric patients is difficult in many ways because the health care providers and parents face many challenges not experienced, or experienced to a lesser extent, than when medications are prescribed for and taken by adults. Firstly, less information is available about the use of most medications for pediatric

Correspondence to: Md. Saiful Islam Pathan; E-mail: sip@ sub.edu.bd

DOI: https://doi.org/10.3329/bpj.v22i2.42316 
patients. Secondly, many drugs that are used for some pediatric patients are not in appropriate dosage forms for use by children (Sagraves, 2006). A major concern is at what age children can safely swallow solid oral dosage forms like tablets or capsules is not clearly defined. Thus, resulted the need for diverse solid oral dosage forms for pediatric population (Hideaki et al., 2008). Oral thin films are novel drug delivery systems especially suitable for both pediatric and geriatric patients who experience difficulties in swallowing traditional solid oral dosage forms (Committee for medicinal and products for human use, 2006). Most of the pharmaceutical companies have directed their search activity in developing viable dosage alternatives from oral route for pediatrics, geriatric, noncompliant or nauseous patients. Research in the oral drug delivery segment has led to evolution of dosage forms from simple conventional tablets/capsules to modified release tablets/capsules to oral disintegrating tablet to wafer to the recent development of fast dissolving oral films. Fast dissolving oral film is a novel drug delivery system for the oral delivery of the drugs prepared using different hydrophilic polymers that rapidly dissolves on the top or the floor of the tongue or buccal cavity. The delivery system consists of a very thin oral strip, which is simply placed on the patient's tongue or any oral mucosal tissue, instantly wet by saliva the film rapidly hydrates and adheres onto the site of application. It then rapidly disintegrates in a matter of seconds and dissolves to release medication for oromucosal absorption. Today, fast dissolving oral films are a well proven and worldwide accepted technology for the systemic delivery of active pharmaceutical ingredients (Pathi et al., 2012).

Palonosetron is a tricyclic 5- $\mathrm{HT}_{3}$ receptor antagonist used as an anti-emetic, and has prophylactic and/or therapeutic effects on nausea and/or vomiting. In particular, palonosetron is useful as an anti-emetic against for vomiting that occurs after surgery, chemotherapy, and radiotherapy. Palonosetron has a chemical name of (3aS)-2-[(3S)1-azabicyclo[2.2.2] oct-3-yl]-2,3,3a,4,5,6-hexahydro1H-benz[de]isoquinolin-1-one $\left(\mathrm{C}_{19} \mathrm{H}_{24} \mathrm{~N}_{2} \mathrm{O}\right)$ and a molecular weight of $296.407 \mathrm{~g} / \mathrm{mol}$ (Yoon et al., 2014).

\section{Materials and Methods}

Materials: Palonosetron hydrochloride was gift sample from Shanghai Jinhe Bio-Technology Co., Ltd. Polymer A was obtained from BASF, Germany and plasticizer $\mathrm{B}$ was obtained from Unilever Bangladesh Ltd. Citric acid was purchased from Merck, India. Ethanol was from Merck, Germany. The rest of all other chemicals and reagents used were analytical grade.

Preparation of Palonosetron hydrochloride oral thin film: Table 1 shows the detailed composition of the oral thin film of palonosetron hydrochloride formulations which were used in the present study.

Calculation of drug quantity: For $68 \mathrm{~mm}$ x 68 $\mathrm{mm}$ rectangular glass plate, the total area of glass plate $=68 \times 68=4624 \mathrm{~mm}^{2}$; size of the film $=10 \mathrm{~mm}$ $\mathrm{x} 10 \mathrm{~mm}=100 \mathrm{~mm}^{2}$; so, theoretically total number of film $=4624 / 100=46.24$; Total quantity of drug $(0.5$ $\mathrm{mg}$ ) should be taken in one batch = about $26 \mathrm{mg}$ including $1 \%$ overage of API.

Preparation: Polymer was taken in a measured quantity of solvent and mixed for $30 \mathrm{~min}$ with magnetic stirrer to dissolve. To this solution, plasticizer B was added with continuous stirring and mixed for 20 min with magnetic stirrer. After that, citric acid dissolved in solvent was added with continuous stirring and mixed for 20 min with magnetic stirrer to dissolve the contents. To the base solution, firstly the API and secondly aspartame were added with continuous stirring and mixed for $20 \mathrm{~min}$ with continuous stirring to dissolve properly. The solution was sonicated for $1 \mathrm{~min}$ to remove bubbles. The final film solution was then casted on glass plate and dried in oven at $60^{\circ} \mathrm{C}$ about $3 \mathrm{hr}$ or until dry. Finally peeled out the film easily from glass plate and cut the films into strips of desired dimensions with a stainless-steel cutter and then packed in a highdensity polyethylene sheet, sealed and stored in desiccators at room temperature. 


\section{Evaluation of oral thin film of palonosetron hydrochloride}

Weight variation (Sultana et al., 2013): The weight of the films was determined by using analytical balance. When manufacturing the oral films, the film solutions were cast into sheets and then cut into smaller strips of $1 \mathrm{~cm}^{2}(1 \mathrm{~cm} \times 1 \mathrm{~cm})$. Oral films were cut from different sheets and the variability between the respective polymers as well as the variability between the polymers was investigated. The results are shown in table 2 .

Thickness (Sultana et al., 2013): The thickness of film was measured by micrometer screw gauge at different strategic locations. Each film was measured at 5 positions (centre and four corners) and the mean thickness was calculated. This is essential to determine uniformity in the thickness of the film. Thickness uniformity is directly related to the dose accuracy of the film. The results are shown in table 2 .

Folding endurance (Sultana et al., 2013): Folding endurance was determined by repeated folding of the film in the same position place until breaking of the film. The times the film is folded without breaking was computed as the folding endurance value. The values have been shown in results and discussion.

Table 1. Composition of various formulations of palonosetron hydrochloride oral thin film

\begin{tabular}{lccccccccc}
\hline & \multicolumn{1}{c}{ Formulation code } \\
\cline { 2 - 9 } Name of the ingredients & F1 & F2 & F3 & F4 & F5 & F6 & F7 & F8 & F9 \\
\hline Palonosetron hydrochloride (mg) & 0.5 & 0.5 & 0.5 & 0.5 & 0.5 & 0.5 & 0.5 & 0.5 & 0.5 \\
Polymer A (mg) & 13 & 14 & 15 & 16 & 17 & 18 & 19 & 20 & 21 \\
Plasticizer B (mg) & 2 & 2 & 2 & 4 & 4 & 4 & 6 & 6 & 6 \\
Citric acid (mg) & 0.2 & 0.2 & 0.2 & 0.2 & 0.2 & 0.2 & 0.2 & 0.2 & 0.2 \\
Aspartame (mg) & 0.8 & 0.8 & 0.8 & 0.8 & 0.8 & 0.8 & 0.8 & 0.8 & 0.8 \\
Solvent (Ethanol-Water) (1:1) (ml) & 0.2 & 0.2 & 0.2 & 0.2 & 0.2 & 0.2 & 0.2 & 0.2 & 0.2 \\
\hline
\end{tabular}

Table 2. Evaluation of the palonosetron hydrochloride oral thin film.

\begin{tabular}{ccccc}
\hline Code & Weight variation $(\mathrm{mg})$ & Thickness $(\mathrm{mm})$ & Content uniformity $(\%)$ & DT $(\mathrm{Sec})$ \\
\hline F1 & $14.33 \pm 0.13$ & $0.098 \pm .0016$ & $97.7 \pm 1.2$ & 25 \\
F2 & $15.29 \pm 0.15$ & $0.107 \pm .0015$ & $98.7 \pm 1.5$ & 28 \\
F3 & $16.23 \pm 0.13$ & $0.115 \pm .0028$ & $97.3 \pm 0.99$ & 30 \\
F4 & $17.26 \pm 0.18$ & $0.125 \pm .0021$ & $97.1 \pm 1.37$ & 32 \\
F5 & $18.24 \pm 0.17$ & $0.132 \pm .0021$ & $97.6 \pm 1.06$ & 34 \\
F6 & $19.25 \pm 0.16$ & $0.141 \pm .0024$ & $98.6 \pm 1.44$ & 36 \\
F7 & $20.27 \pm 0.09$ & $0.150 \pm .0019$ & $98.5 \pm 0.90$ & 38 \\
F8 & $21.25 \pm 0.14$ & $0.159 \pm .0018$ & $98.1 \pm 1.15$ & 42 \\
F9 & $22.27 \pm 0.18$ & $0.165 \pm .0025$ & $96.8 \pm 1.81$ & 44 \\
\hline
\end{tabular}

All the values are expressed as mean $\pm \mathrm{SD}, \mathrm{n}=10$; DT $=$ disintegration time.

Determination of $p H$ value (Sultana et al., 2013): The $\mathrm{pH}$ value was determined by dissolving one oral film in $2 \mathrm{ml}$ distilled water and measuring the $\mathrm{pH}$ of the obtained solution by using $\mathrm{pH}$ paper.
Differences were expected because various polymers were used as well as the addition of API.

Disintegration testing (Sultana et al., 2013): In vitro disintegration time was determined visually in a 
glass beaker of $25 \mathrm{ml}$ distilled water with swirling every $10 \mathrm{sec}$. The disintegration time is the time when the film starts to break or disintegrates. The results are shown in table 2.

Content uniformity (Sultana et al., 2013): The content uniformity test was used to ensure that every film contains the intended amount of drug substance with little variation among films within a film. Six pieces, each $1 \mathrm{~cm}^{2}(1 \times 1 \mathrm{~cm})$, were cut from the whole film, and analyzed for drug content using HPLC method at the wavelength of $242 \mathrm{~nm}$. Same procedure was repeated for all the nine batches. The results are shown in table 2.

In vitro dissolution (Sultana et al., 2013): The in vitro drug release of film was studied by using a USP 23 type 2 rotating paddle dissolution test apparatus.
For this, $500 \mathrm{ml}$ phosphate buffer ( $\mathrm{pH}$ 6.8) was used and kept at $37 \pm 5^{\circ} \mathrm{C}$ while the basket was set at 50 rpm. A film sample of $1 \mathrm{~cm}^{2}(1 \mathrm{~cm} \times 1 \mathrm{~cm})$ was fixed onto the specially designed SS disk with the help of cyanoacrylate adhesive. The disk was put at the bottom of the dissolution vessel so that the film remained on the upper side of the disk. Five millilitres of samples were taken at interval of 60 second and the same amount was replaced with fresh buffer. The withdrawn samples were filtered through Whatman filter paper and analyzed using a spectrophotometer at a wavelength of $256 \mathrm{~nm}$. The cumulative percentage release for different formulations was calculated. The relationship between time and percentage release were plotted as shown in figures 1 and 2 .

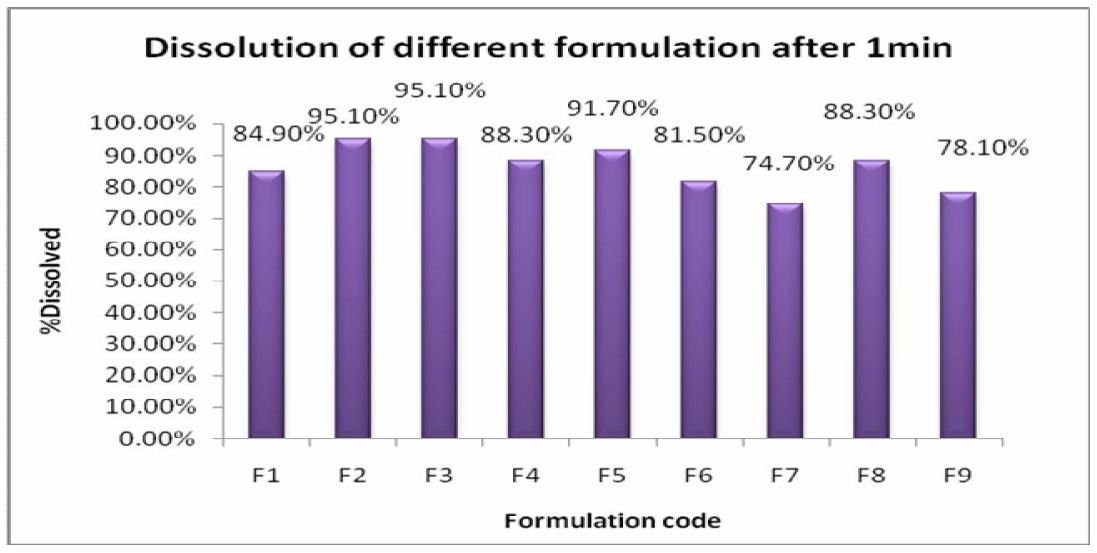

Figure 1. In vitro drug release of different formulations from $\mathrm{F} 1$ to $\mathrm{F} 9$ of palonosetron hydrochloride oral thin film (after $1 \mathrm{~min}$ ).

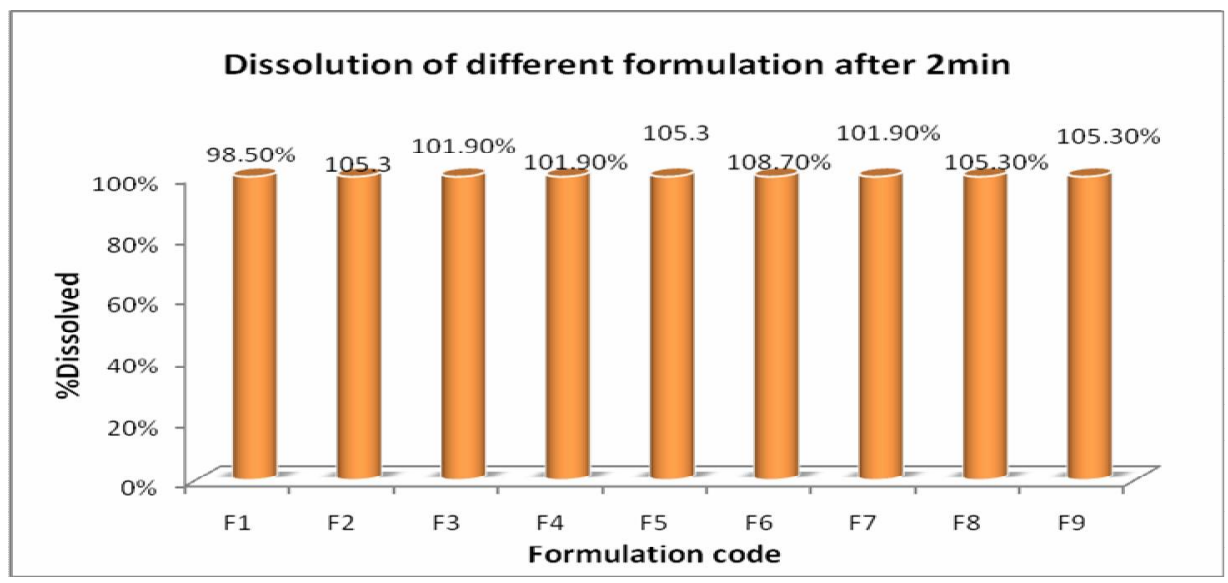

Figure 2. In vitro drug release of different formulation from F1 to F9 of palonosetron hydrochloride oral thin film (after 2 min). 
FTIR (ATR) study of sample: FTIR spectrophotometer was used to determine drugexcipients compatibility by using pure palonosetron hydrochloride and physical mixtures of palonosetron hydrochloride and excipients. The IR spectra were determined by the ATR method between $2000 \mathrm{~cm}^{-1}$ and $650 \mathrm{~cm}^{-1}$. FTIR studies revealed that there was no interaction of the IR bands of palonosetron hydrochloride as shown in the figures 3 and 4 .

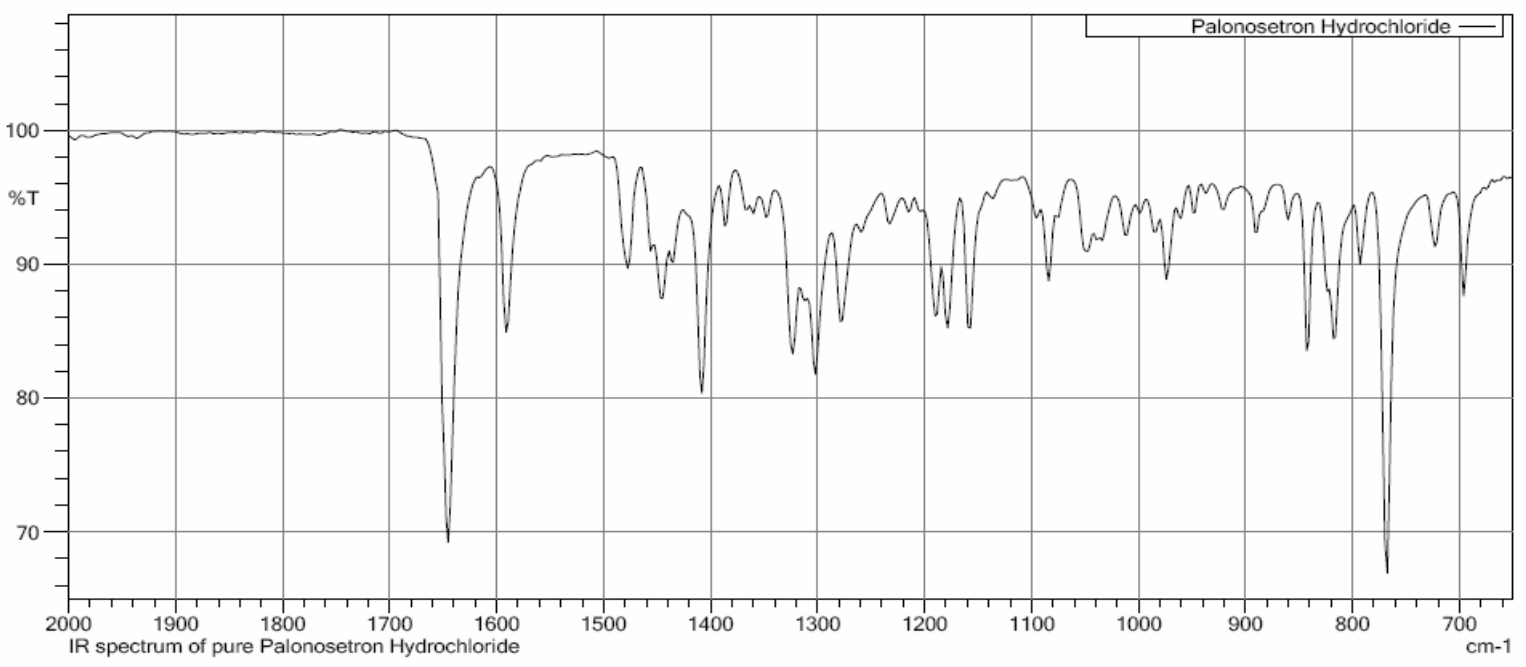

Figure 3. FTIR spectra of pure palonosetron hydrochloride

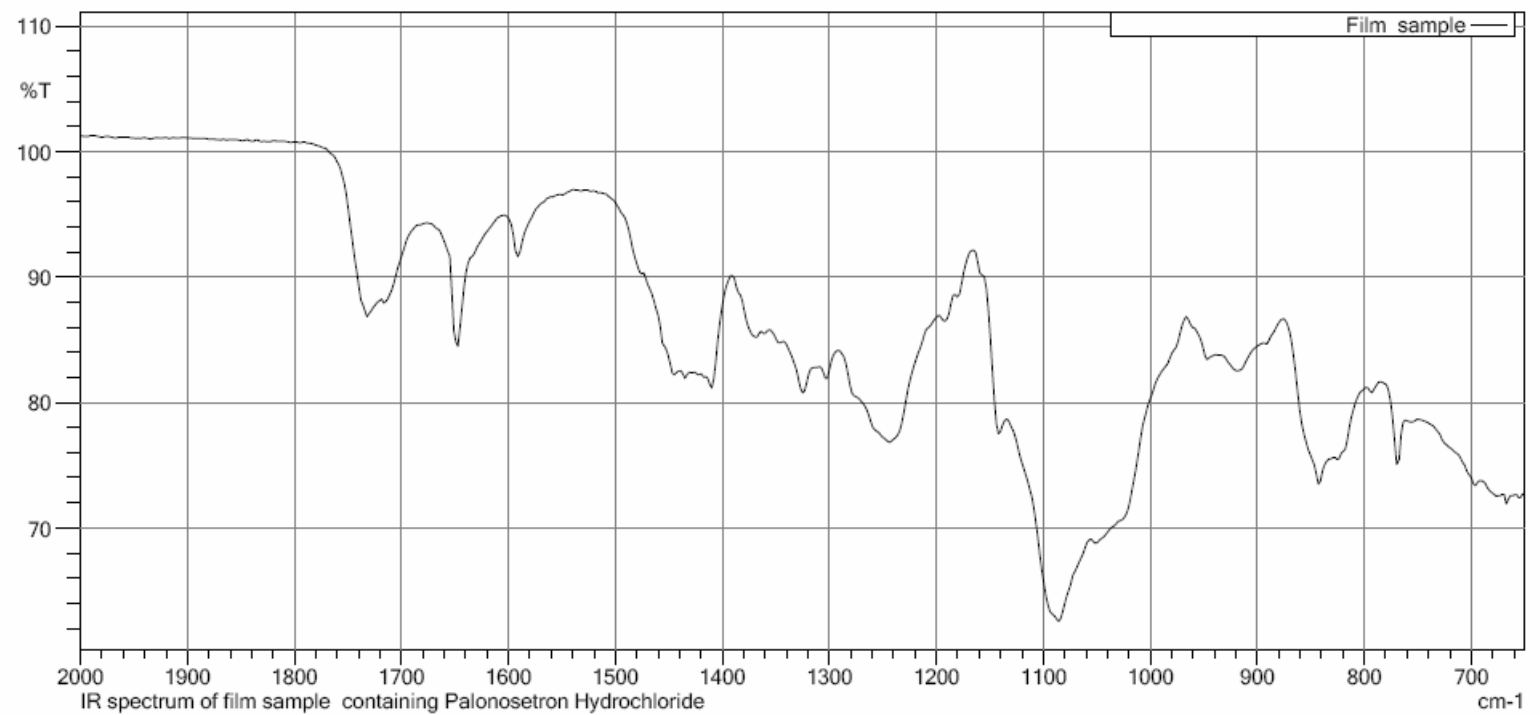

Figure 4. FTIR spectra of mixtures of palonosetron hydrochloride and excipients.

HPLC study of Palonosetron oral thin film: HPLC or High-performance Liquid Chromatography was used to identify and quantify Palonosetron $\mathrm{HCl}$ present in the oral film by comparing with the standard sample of palonosetron hydrochloride at wavelength of $242 \mathrm{~nm}$ in UV detector. The retention time for Palonosetron hydrochloride was about 4.4 min both in standard and sample solution using octadecyl silane $\left(\mathrm{C}_{18}\right)$ column. An extra peak was observed before the main peak of Palonosetron 
hydrochloride in case of standard due to presence of diluents. Some additional responses were observed in the sample chromatogram due to diluents and other excipients mixture used in the sample preparation. Peak responses in standard and sample chromatogram were varied due to concentration differences in standard and sample solution. However, the chromatogram of standard and film sample revealed that there was no significant impact on oral thin film sample for HPLC analysis as shown in figures 5,6 and 7 .

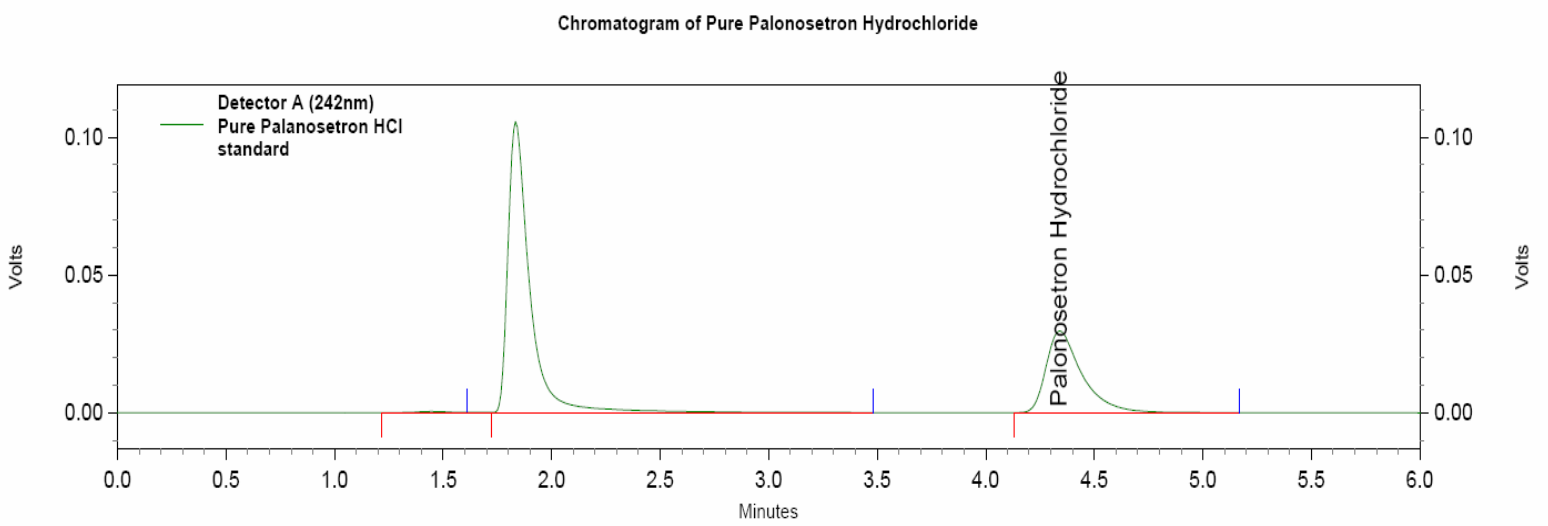

Figure 5. HPLC chromatogram of standard palonosetron $\mathrm{HCl}$.

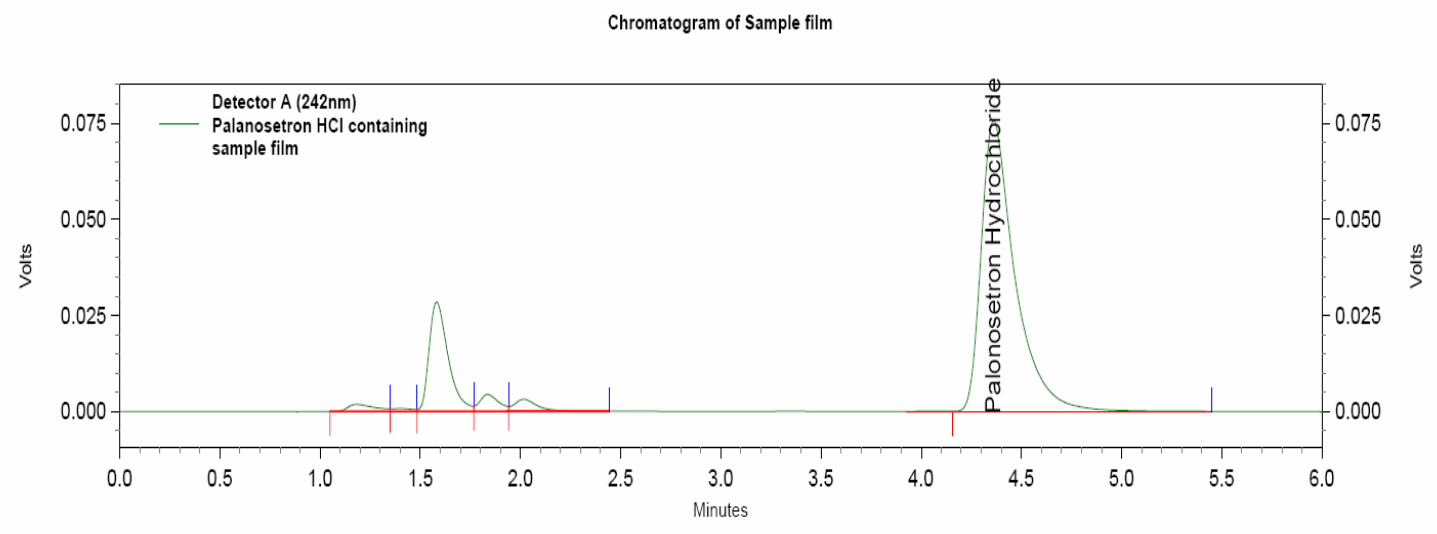

6. HPLC chromatogram of oral thin film containing palonosetron $\mathrm{HCl}$

Chromatogram of Placebo film in HPLC

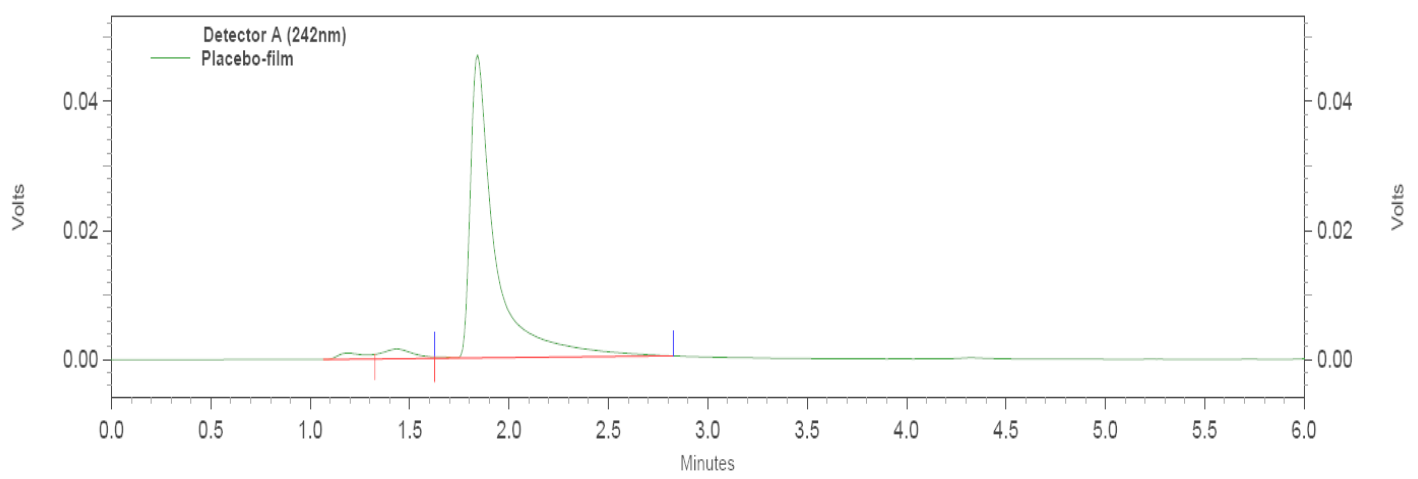

Figure 7. HPLC chromatogram of placebo film. 


\section{Results and Discussion}

In the present study, oral thin films of palonosetron hydrochloride were successfully prepared containing different concentration of polymer and other ingredients using solvent casting method. Mixture of water and ethanol (1:1) was uses as solvent. The drying time was about 3 hrs. The films were evaluated for various properties including thickness, weight variation, $\mathrm{pH}$, folding endurance, percent content uniformity, in vitro drug dissolution as well as drug-excipients interaction. The weights of the films of all batches were acceptable. A very low standard deviation (SD) value indicated that the method used was reproducible and consistent. The folding endurance of all the batches were F1(976), F2(866), F3(855), F4(742), F5(731), F6(712), F7(707), F8(713), F9(705). Batches with higher amount of polymer gave lower folding endurance (table 1). The results of weight and drug content uniformity test showed that the film was homogenous and drug was uniformly distributed in the films. Films having thickness were adequate for handling and use. The disintegration time was good enough. It is also reflected in this study that more or less all the formulations showed good dissolution properties i.e. fast dissolving characters.

\section{Conclusion}

Oral thin films are intended for the application in the oral cavity, which is an innovative and promising dosage form especially for use in pediatrics and geriatrics. The in vitro studies have shown that this is a potential drug delivery system for Palonosetron hydrochloride with a considerably good physicochemical characteristics and release profile. Based on the above evaluations, it is quite evident that the prepared fast dissolving oral thin film of palonosetron hydrochloride containing film forming polymer A and plasticizer B was the best choice which provided faster release of drug within short period of time with better acceptability in a simple, less time consuming and cost effective way. Further study on formulation optimization, drug excipients compatibility, scale up and stability study are needed to confirm the appropriateness of these formulated oral thin films.

\section{References}

Arya, A., Chandra, A., Sharma V. and Pathak. K. 2010. Fast dissolving oral films: an innovative drug delivery system and dosage form. Int. J. Chem. Tech. Res. 2, 576-583.

Chauhan, I., Yasir, M. and Nagar, P. 2012. Insights into polymers: film formers in mouth dissolving films. Drug Invent. Today. 3, 56-73.

Committee for medicinal and products for human use, European medicines agency, EMEA, Reflection paper. 2006. Formulation of choice of the paediatric population, Available from https://www.ema.europa. eu/en/documents/scientific-guideline/reflection-paperformulations-choice-paediatric-population_en.pdf

Dixit, R.P. and Puthli, SP. 2009. Oral strip technology: overview and future potential. J. Control Rel. 139, 94107.

Hideaki, O., Eiji, S., Yusaku, S., Kaisuke, Y., Yasunari, T., Masaru, H., Eiji, N., Kaname, N., Toru, S., Mikoto, B. and Saitoh, E. 2008. Development of an easily swallowed film formulation. Int. J. Pharm. 35, 62-66.

Pathi, P.J. and Raju, N.A. 2012. The estimation of palonosetron hydrochloride in parenterals by RPHPLC. Asian J. Pharm. Tech. 2, 77-79.

Sagraves, R. 2006. Pediatric Dosing and Dosage Forms. Encyclopedia of Pharmaceutical Technology, 3rd edition.

Sultana, F., Arafat, M and Pathan, S.I. 2013. Preparation and evaluation of fast dissolving oral thin film of caffeine; Int. J. Pharm. Biol. Sci. 3, 153-161.

Yoon, H.J. and Lee, S.J. 2014. Pharmaceutical composition containing palonosetron Available from https://patents.google.com/patent/US9877959B2/en. 